\title{
The JAK2 V617F Mutation in a Patient with Acute Myeloid Leukemia: a Case Report and Literature Review
}

Jia Li and Hongtao Wang*

Department of Hematology, Shengjing Hospital, China Medical University, Shenyang, PR China

\begin{abstract}
Acute myeloid leukemia (AML) is a malignant clonal disease of hematopoietic stem cells. It can also be secondary to other blood system diseases such as myelodysplastic syndromes (MDS) and myeloproliferative tumors neoplasms (MPN). The JAK2 V617F mutation has been reported in MPN, but rarely reported in patients with AML. This report is related a 60 -year-old patient who suffered from AML with JAK2 V617F mutation. The platelet count was $603 \times$ $109 / \mathrm{L}$ at onset. He was given the DA regimen for induction chemotherapy and received complete remission. But the regimen was forced to stop due to acute cerebral infarction after second HDA regimen consolidation chemotherapy. Six months later, the patient had a recurrence. No complete remission was achieved after repeated chemotherapy. At the same time, the patient was given aspirin for antiplatelet aggregation and interferon to reduce platelets, but he stopped using interferon because of the severe infection. Later the patient presented with acute cerebral infarction again. This is a rare case report indicating that the patient with JAK2 V617F mutation had a poor prognosis and had recurrent thrombotic diseases because of elevated platelets.
\end{abstract}

Keywords: Acute myeloid leukemia; JAK2 V617F

\section{Introduction}

Acute myeloid leukemia (AML) is a malignant clonal disease of hematopoietic stem cells. AML is derived from malignant transformation of hematopoietic stem cells during the process of cell differentiation and development, at the same time, it can also be secondary to chemotherapy and radiotherapy of tumor related diseases or other blood system diseases such as myelodysplastic syndromes (MDS) and myeloproliferative tumors neoplasms (MPN) [1]. We report a rare case of a patient with JAK2 V617F positive mutation in $\mathrm{AML}$ and review the relevant literature to investigate the pathogenesis and prognosis of AML associated with JAK2 V617F mutation.

\section{Case History and Clinical Information}

The patient was a 60 -year-old man who complained with "limbs pain for one week". He was admitted to our hospital on January 21, 2015. The present blood analysis indicated as follows: white blood cell (WBC) count, $2.1 \times 10^{9} / \mathrm{L}$; hemoglobin $(\mathrm{Hb}), 102 \mathrm{~g} / \mathrm{L}$; platelet count, $603 \times 10^{9} / \mathrm{L}$. Coagulation function: prothrombin time, 12.7 seconds, activated partial thromboplastin time, 42 seconds, fibrinogen content, $4.0 \mathrm{~g} / \mathrm{L}, \mathrm{D}$-dimer $110 \mu \mathrm{g} / \mathrm{L}$. Bone marrow morphology (Figure 1): the proliferation of bone marrow was active. The primary granulocytes (type I+II) accounted for $36.40 \%$; the red cell proliferation was active;

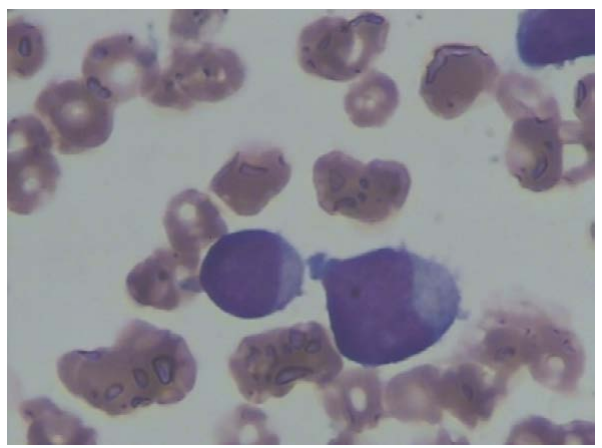

Figure 1: Bone marrow morphology in January 21, $2015(\mathrm{He} \times 1000)$ the abnormal hyperplasia of the mononuclear system accounted for $15.20 \%$ (ANC), $20.88 \%$ (NEC). The WT1 gene and the JAK2 V617F gene were positive. The results of flow cytometry (FCM) showed abnormal immature myeloid cells accounted for $21.52 \%$. CD45, CD34, CD117, HLA-DR, CD38, CD13 were expressed, some cells expressed CD33, but CD10, CD19, CD7, CD2, CD9, CD56, CD123, CD15, CD11b, CD4, CD64, CD14 were negative. Karyotype analysis was normal. The patient was eventually diagnosed with AML-M4a. From January 29, 2015, the patient received the DA regimen for induction chemotherapy as follows: $\mathrm{D}$ (daunorubicin) $60 \mathrm{mg} /$ day, $\mathrm{VD}, \mathrm{d} 1-\mathrm{d} 3$; Ara-C (cytosine arabinoside) $200 \mathrm{mg} /$ day, VD, d1-d7. After 3 weeks, the patient received complete remission. From March 6, 2015, the patient was given HDA regimen for consolidation chemotherapy as follows: D (daunorubicin) $60 \mathrm{mg} / \mathrm{day}, \mathrm{VD}, \mathrm{d} 1-\mathrm{d} 3$; D (daunorubicin) $40 \mathrm{mg} /$ day, d4; Ara-C (cytosine arabinoside) $300 \mathrm{mg} /$ day, VD, d1-d7; $\mathrm{H}$ (homoharringtonine) $2 \mathrm{mg} / \mathrm{day}, \mathrm{d} 1-\mathrm{d} 5.21$ days after chemotherapy, the patient suffered from mental disorder and dysfunction of the right limb. So he was admitted to the neurology department in our hospital. The blood routine and coagulation function were not obviously abnormal. He received head MRI, showing that there existed recent infarction of the left cerebellum and pons. MRA: no obvious abnormality. MRV: the left transverse sinus and sigmoid sinus showed unclear part (Figure 2). He was given butyphthalide $100 \mathrm{~mL}$ two times a day, aspirin $100 \mathrm{mg}$ once a day and clopidogrel $75 \mathrm{mg}$ once a day. The condition of the patient was better than before, but still left the enunciation unclear and dysfunction of the right limb. Six months

*Corresponding author: Wang $\mathrm{H}$, Department of Hematology, Shengjing Hospital, China Medical University, Shenyang, PR China, Tel: 862423256666 ; E-mail: wanght@sj-hospital.org

Received September 08, 2017; Accepted September 15, 2017; Published September 28, 2017

Citation: Li J, Wang H (2017) The JAK2 V617F Mutation in a Patient with Acute Myeloid Leukemia: a Case Report and Literature Review. J Blood Lymph 7: 177. doi: 10.4172/2165-7831.1000177

Copyright: ( $2017 \mathrm{Li} \mathrm{J}$, et al. This is an open-access article distributed under the terms of the Creative Commons Attribution License, which permits unrestricted use, distribution, and reproduction in any medium, provided the original author and source are credited. 


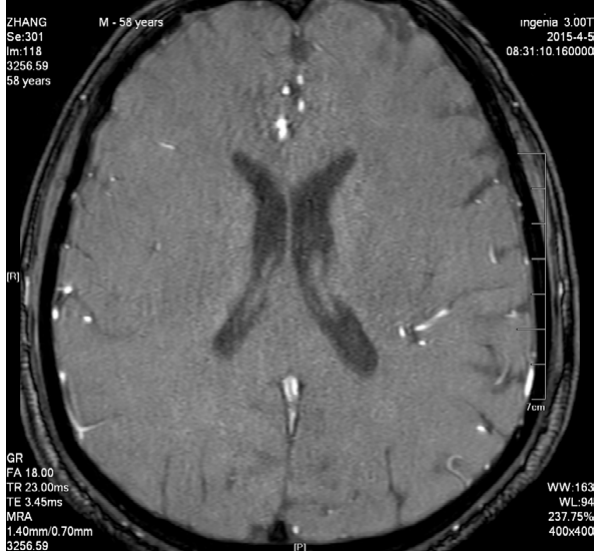

Figure 2: Magnetic resonance imaging (MRI) scan in April 5, 2015.

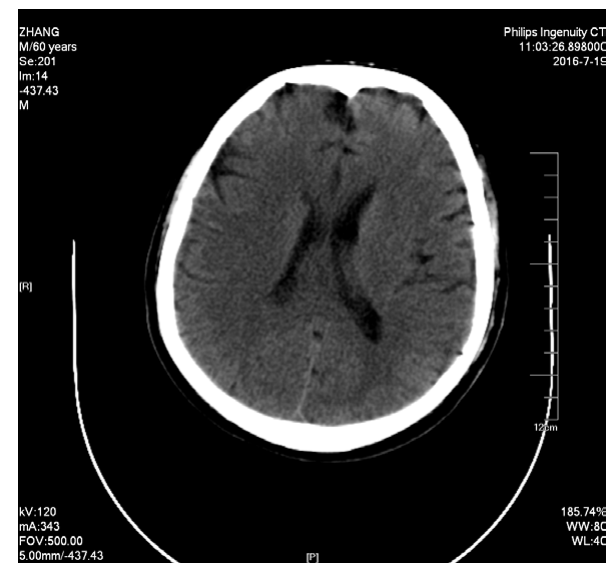

Figure 3: Cranial computer tomography (CT) scan in July 19, 2016.

later, the blood analysis indicated as follows: white blood cell (WBC) count $1.7 \times 10^{9} / \mathrm{L}$; hemoglobin $(\mathrm{Hb}), 60 \mathrm{~g} / \mathrm{L}$; platelet count, $704 \times 10^{9} / \mathrm{L}$. Coagulation function: prothrombin time, 11.2 seconds, activated partial thromboplastin time, 33.3 seconds, fibrinogen content, $3.03 \mathrm{~g} / \mathrm{L}$, $\mathrm{D}$-dimer $48 \mu \mathrm{g} / \mathrm{L}$. Bone marrow morphology suggested a recurrence of the disease. The patient was treated with DA regimen for two times, $\mathrm{CAG}$ regimen once, and MA regimen once. No complete remission was achieved. At the same time, the patient was given aspirin $100 \mathrm{mg}$ once a day for antiplatelet aggregation during chemotherapy, and the recombinant human interferon alpha-2b 3 million IU was injected subcutaneously every other day to reduce platelets. The patient was asked to stop using interferon because of the severe infection with interferon. From June 17, 2016, the patient was given DA regimen again. 30 days after the chemotherapy, the patient suffered from mental disorder again, performing as nausea and vomiting, disturbance of consciousness. During this time, his blood analysis was as follows: white blood cell (WBC) count, $1.9 \times 10^{9} / \mathrm{L}$; Hemoglobin $(\mathrm{Hb}), 61 \mathrm{~g} / \mathrm{L}$; platelet count, $974 \times 10^{9} / \mathrm{L}$. Coagulation function: prothrombin time, 13.7 seconds, activated partial thromboplastin time, 36.9 seconds, fibrinogen content, $5.13 \mathrm{~g} / \mathrm{L}$, D-dimer $508 \mu \mathrm{g} / \mathrm{L}$. He received head CT showing that there existed a hypodense shadow in the left cerebellum, and small slices of hypodense shadows in the brainstem (Figure 3). He presented with acute cerebral infarction again. The abdominal CT showed that the wall was thickened of splenic flexure of the colon, with localized peritonitis in the left abdomen, inflammation?
Intestinal obstruction? We gave the patient aspirin $100 \mathrm{mg}$ once a day for antiplatelet aggregation, and butyphthalide twice daily to improve circulation, along with calf blood protein to nutrite brain cells. At the same time, we gave him gastrointestinal decompression to treat intestinal obstruction. Later, the patient's condition was stable; the patient was discharged from the hospital.

\section{Discussion}

The JAK protein family is a kind of non-receptor tyrosine kinase. Four members have been identified, they are JAK1, JAK2, JAK3 and TYK2, respectively [2]. Kralovics first reported the discovery of JAK point mutation in the bone marrow proliferative tumor (MPN) in 2005, which is closely related to the occurrence of MPN disease [3]. The self-restraint function of JAK2 V617F gene encoded protein kinase $\mathrm{JH} 2$ region was released, the downstream STAT family protein was continuously activated to increase transcription and translation of the target genes related to proliferation, and the activation of MAPK pathway cooperatively regulated the formation of erythroid colony formation through the cross regulation of PI3K signal pathway [4]. However, some studies indicated that JAK2 V617F mutation could appear in AML. The mutation rate of JAK V617F in patients with primary AML in a German study with a large sample was $1.1 \%$, and the mutation rate was $2.7 \%$ in Korea research center. Because of its relatively low mutation rate, there is no clear explanation for the prognosis of AML [5-8]. In this case, the patient was completely relieved for the first time, but did not receive secondary remission after recurrence. Studies have shown that JAK2 gene mutation in AML cells have poor sensitivity to chemotherapy, and AML patients with a high level of phosphorylation of the JAK2 gene have poor prognosis and short survival time [9]. In addition, JAK2 gene mutation was associated with thrombotic events. JAK2 V617F mutation can activate platelets, leukocytes and blood coagulation cascade reaction, and produce platelet aggregation in endothelial injury, which leads to the formation of arteriovenous thrombosis [10]. The risk of thromboembolic disease in the patients with JAK2 V617F positive was higher than that of JAK2 V617F negative patients. In this case, aspirin was used to prevent platelet aggregation during chemotherapy, but interferon was not tolerated by patient because of severe infection. The mutation of JAK2 V617F gene in MPN patients result in hyperphosphorylation of JAK2, interferon can regulate JAK2 signal pathway by reducing the copy number of JAK2V617F in MPN patients [11]. JAK2 inhibitor Ruxolitinib has inhibitory effect on both wild-type and mutant JAK2. After the application of Ruxolitinib on the hematopoietic stem/ progenitor cells cultured in vitro of patients with polycythemia vera, the number of erythroid colony formation was significantly reduced. The dose-dependent apoptosis and the decrease of the phosphorylation of JAK2 in the downstream sites were observed. The excessive STAT3 signal can be normalized. It can also significantly inhibit inflammatory cytokines and some angiogenesis and fibroblast growth factors such as vascular endothelial growth factor (VEGF) and basic fibroblast growth factor [12,13]. In this case, the patient with JAK2 mutation had a thrombotic disease due to increased platelet count. If the patient can regularly use interferon or JAK2 inhibitors during chemotherapy, he may be having a better prognosis. We need to accumulate more clinical data to explore the efficacy and prognosis.

\section{References}

1. Rampal R, Mascarenhas J (2014) Pathogenesis and management of acute myeloid leukemia that has evolved from a myeloproliferative neoplasm. Cur Opin Hematol 21: 65-71. 
Citation: Li J, Wang H (2017) The JAK2 V617F Mutation in a Patient with Acute Myeloid Leukemia: a Case Report and Literature Review. J Blood Lymph 7: 177. doi: 10.4172/2165-7831.1000177

Page 3 of 3

2. Seavey MM, Dobrzanski P (2012) The many faces of Janus kinase. Biochem Pharmacol 83: 1136-1145.

3. Kralovics R, Passamonti F, Buser AS, Teo SS, Tiedt R, et al. (2005) A Gain-ofFunction Mutation of JAK2 in Myeloproliferative Disorders-NEJM. New England Journal of Medicine 352: 1779-1790.

4. Wolf A, Eulenfeld R, Gabler K, Rolvering C, Haan S, et al. (2013) JAK2-V617Finduced MAPK activity is regulated by PI3K and acts synergistically with PI3K on the proliferation of JAK2-V617F-positive cells. Jak-Stat 2: e24574.

5. Ohanian M, Bueso-Ramos C, Ok CY, Lin P, Patel K et al. (2015) Acute myeloid leukemia with MYC rearrangement and JAK2 V617F mutation. Cancer Genetics 208: 571-574.

6. Illmer T, Schaich M, Ehninger G, Thiede C (2007) Tyrosine kinase mutations of JAK2 are rare events in AML but influence prognosis of patients with CBFleukemias. Haematologica 92:137-138.

7. Lee JW, Kim YG, Soung YH, Han KJ, Kim SY, et al. (2006) The JAK2 V617F mutation in de novo acute myelogenous leukemias. Oncogene 25: 1434-1436.

8. Wang S, Yan J, Zhou G, Heintzelman R, Hou JS (2016) Myeloproliferative Neoplasm or Reactive Process? A Rare Case of Acute Myeloid Leukemia and
Transient Posttreatment Megakaryocytic Hyperplasia with JAK-2 Mutation. Case Reports in Hematology 2016: 1-5.9.

9. Ikezoe T, Kojima S, Furihata M, Yang J, Nishioka C, et al. (2011) Expression of p-JAK2 predicts clinical outcome and is a potential molecular target of acute myelogenous leukemia. International Journal of Cancer 129: 2512-2521.

10. AM Vladareanu, Popov V, Bumbea H, Onisai M, Ilea A, et al. (2011) Splanchnic vein thrombosis, the onset manifestation in JAK positive Chronic Myeloproliferative Disorders Neoplasms. Journal of Medicine \& Life 4: 97-101.

11. Fu JZ, Xu Q, Zhao YL (2015) Anti-angiogenic effect of interferon on JAK2 V617F positive myeloproliferative neoplasma and its anti-angiogenic mechanisms. Natl Med J China 95: 3727-3732.

12. Verstovsek S, Mesa RA, Gotlib J, Levy RS, Gupta V, et al. (2015) Efficacy, safety, and survival with ruxolitinib in patients with myelofibrosis: results of a median 3-year follow-up of COMFORT-I. Haematologica 100: 479-488.

13. Szymanska J, Smolewski P, Majchrzak A, Cebula-Obrzut B, Chojnowski K, et al. (2015) Pro-Apoptotic Activity of Ruxolitinib Alone and in Combination with Hydroxyurea, Busulphan, and PI3K/mTOR Inhibitors in JAK2-Positive Human Cell Lines. Advances in Clinical and Experimental Medicine 24:195-202. 